\title{
Pathologische Frakturen bei Osteoarthropathie am Beispiel der diabetisch-neuropathischen Osteoarthropathie
}

\author{
■ Torsten Eyfferth, Hans-Peter Abt, Reinhard Hoffmann
}

\section{Zusammenfassung}

Osteoarthropathien mit den im Verlauf teils schwerwiegenden Destruktionen der betroffenen Knochen und Gelenke treten häufig infolge einer langjährigen diabetischen Stoffwechselerkrankung bei gleichzeitig progredienter Polyneuropathie auf. Insbesondere im Bereich des Sprunggelenks und des Fußes verursachen die veränderte Statik und Belastungsfähigkeit in Verbindung mit einer erhöhten Verletzungsanfälligkeit der Haut im Sinne von plantaren Ulzerationen erhebliche Behandlungsprobleme. Die Behandlungsmöglichkeiten sind begrenzt und zunächst konservativ ausgerichtet. Das Therapiekonzept umfasst vorübergehende Immobilisationen oder Entlastungen der betroffenen Gliedmaßen, die Versorgung mit orthopädischem Maßschuhwerk bzw. auch mit Orthesen. Der fortschreitende Knochenumbauprozess macht ständige Anpassungen der orthopädischen Hilfsmittel erforderlich. Erst in den späteren Stadien der Erkrankung kommen rekonstruktive Eingriffe zum Tragen.
Pathologic Fractures due to Osteoarthropathy: The Example of Diabetic Neuropathic Osteoarthropathy

Osteoarthropathy with a development of sometimes severe destruction of the concerned joints usually appears due to a long-term diabetic metabolic disease along with a progressing polyneuropathy. Especially with regard to the ankle and the foot, the changed statics und ability to bear weight in combination with the heightened vulnerability of the skin for plantar ulcerations cause considerable problems in the treatment. The possibilities of treatment are limited and at first of a conservative nature. The concept of therapy consists of temporary immobilisation or relief of the concerned extremity, application of orthopaedic custom-made shoes or an orthotic device. The progress changes of the bone make it necessary to continuously adjust these orthopaedic aids. Only in a later stages of the disease are reconstructive surgical interventions performed.

\section{Einleitung}

Die Osteoarthropathie (Synonym: Charcot joint, Neuroarthropathia diabetica, Rocker foot) haben Jean Marie Charcot und Féré 1883 bei der Tabes dorsalis, dem Endstadium der Syphilis, erstmals beschrieben. Der Begriff des Charcot-Fußes findet vor allem in der englisch- und französischsprachigen Literatur noch immer weite Verbreitung.

Als Spätfolgen einer diabetischen Neuropathie hat sie Jordan erst 50 Jahre später im Jahr 1936 in Verbindung gebracht und

OP-JOURNAL 2006; 22: 46-51

(c) Georg Thieme Verlag KG Stuttgart · New York beschrieben, da durch Entdeckung des Insulins durch den Orthopäden Banting 1921 ein höheres Lebensalter der Diabetiker möglich wurde. Weitere Veröffentlichungen folgten in den Jahren 1942 (Bailey) und 1947 (Root).

Aktuell geworden ist das Thema aber erst in den letzten 20 Jahren. Die Ursache liegt wohl weniger in der Zunahme der Diabetiker als in dem Ansinnen, einen gefühllosen und schwer deformierten Diabetesfuß möglichst lange zu erhalten.

Der ursächliche Entstehungsprozess wurzelt im Wesentlichen auf der diabetischen Neuropathie, wobei durch eine Schädigung des sympathischen Nerven- systems eine gesteigerte periphere Zirkulation und Knochenabsorption resultiert. Im Weiteren handelt es sich um eine Reaktionskette aus Mikro- und Makrotraumata, Abschwächung der Schutzund statokinetischen Reflexe, Bandinstabilitäten sowie vaskuläre Störungen, die in letzter Zeit zunehmend Beachtung finden. Die progrediente Knochenreabsorption (Osteolyse) stellt mit einem fortschreitenden Stabilitätsverlust und dem sukzessiven, schmerzlosen Zusammenbrechen des Vor- und/oder Rückfußskelettes häufig den Endpunkt eines langen Leidensweges für die Betroffenen dar. Die zugrunde liegenden biomechanischen und -chemischen Pathomechanismen dieser sicher multifaktoriell bedingten Erkrankung sind im Wesentlichen noch ungeklärt.

Tab. 1 Synonyme der Osteoarthropathie

- Diabetische Osteoarthropathie

- Diabetikergelenk

- Neuroarthropathia diabetica

- Charcot joint

- Rocker foot

- Diabetic arthropathy

- Multilating arthropathy

\section{Prävalenz und Epidemiologie}

Die Prävalenz der diabetisch-neuropathischen Osteoarthropathie (DNOAP) wird je nach Literaturangabe zwischen 0,15 und $13 \%$ angenommen. Die Erkrankung trifft in zunehmendem Maß auch jüngere Patienten insbesondere im Verlauf eines schweren, über längere Zeit unzureichend eingestellten Diabetes mellitus. Zwischen dem Auftreten der diabetischen Stoffwechselerkrankungen und Erstmanifestation der Osteoarthropathie vergehen im Durchschnitt ca. 10 Jahre. Oftmals sind eher langjährig unerkannte Typ-IIDiabetiker mit einer Übergewichtigkeit und weniger der gut eingestellte Typ-IDiabetiker (im Schnitt nach ca. 30 Jahren) betroffen. 
Unter epidemiologischen Gesichtspunkten ist in Anbetracht der höheren Lebenserwartung und der darüber hinaus zu beobachtenden Zunahme der diabetischen Stoffwechselerkrankungen in unserer Zivilisationsgesellschaft von einer weiteren Häufung des Krankheitsbildes auszugehen.

Bei der Ursachenforschung der diabetisch-neuropathischen Osteoarthropathie spielt die begleitende Polyneuropathie eine entscheidende Rolle. Wurde zunächst kontrovers eine neurotraumatische bzw. neurovaskuläre Genese diskutiert, so scheinen neueren Untersuchungen nach, bei gestörtem neuronalen Schutzmechanismus, sowohl wiederholte Mikrotraumatisierung der gelenkbildenden Knochen als auch autonome neuropathische Gefäßdysregulationen mit der Folge einer Aktivierung von Osteoklasten den fortschreitenden knochen- und gelenkdestruierenden Prozess zu unterhalten.

Das klinische Erscheinungsbild ist initial gekennzeichnet durch einen fortschreitenden, schmerzlosen, nicht entzündlich bedingten Gelenkerguss mit teils massiver Schwellung des umgebenden Weichteilmantels. Im weiteren Verlauf kommt es aufgrund fortschreitender knochenresorptiver Umbauvorgänge zu grotesken Deformitäten an den betroffenen Gelenken. Im Endstadium lässt sich eine zunehmende knochenverdichtende Sklerosierung erkennen.

Die chirurgische Therapie kann dann erforderlich werden, wenn sich aufgrund der knöchernen Umbauvorgänge chronische Druckulzera mit sekundären Komplikationen wie drohenden Knochendurchspießungen und Weichteil- sowie Knocheninfektionen entwickeln.

Im Gegensatz zur Tabes dorsalis, dem Endstadium der Syphilis, ist der Befall anderer Gelenke oder der oberen Extremitäten äußerst selten. In letzter Zeit werden zunehmende Erkrankungsfälle auch an den Kniegelenken beobachtet, wobei sich dies weniger durch eine wirkliche Häufung der Fälle, sondern eher wegen eines zunehmenden Verständnisses gegenüber dem Erkrankungsbild der Osteoarthropathie und deren Erscheinungsformen erklären lässt.

Grundsätzlich bedarf es bei der Diagnosestellung einer differenzialdiagnostischen Abgrenzung zu posttraumatischen Folgezuständen, peripheren Nervenläsionen, arterielle Verschlusskrankheit,

Tab.2 Stadien der diabetischen Osteoarthropathie nach Eichenholtz

\begin{tabular}{lll} 
Stadium I & Zerstörung & $\begin{array}{l}\text { Osteoporose } \\
\text { Osteolyse, Osteonekrose } \\
\text { Arthropathie } \\
\text { Pathologische Frakturen }\end{array}$ \\
\hline Stadium II & Umbau & Spontane Subluxation und Dislokationen \\
\hline Stadium III & Stabilisierung & $\begin{array}{l}\text { Knochenanbau } \\
\text { Sklerosierungen } \\
\text { Verknöcherungen }\end{array}$ \\
& &
\end{tabular}

Osteomyelitis, Osteoporose, malignen Erkrankungen, Syringomyelie, Amyloidose etc.

\section{Klassifikation}

Stadien der diabetischen Osteoarthropathie nach Eichenholtz

Ein wesentlicher Stellenwert im Hinblick auf die Beurteilung des Aktivitätsgrades einer Osteoarthropathie kommt der radiologischen Diagnostik zu. Eichenholtz differenziert die Erkrankung in 3 Stadien:

\section{Stadium I}

Das Stadium I, auch als aktive Phase bezeichnet, ist gekennzeichnet durch eine diffuse und schmerzlose Weichteil- und Gelenksschwellung und lässt radiologisch intraartikuläre Frakturen sowie Dislokation und osteochondrale Destruktionen erkennen. Bedingt durch die neurogenen Sensibilitätsstörungen führt die fortgesetzte Belastung zu fortschreitenden Mikrotraumatisierung mit der Folge entzündlicher Reizzustände. Es resultieren zunehmende Fehlstellungen aufgrund von Ermüdungsfrakturen, die im Rückfuß zu ausgedehnten Destruktionen (Osteonekrose) und Zerfall des spongiösen Knochens von Talus und Kalkaneus führen können. Szintigraphisch können deutliche Mehranreicherungen im Sinne eines erhöhten Knochenstoffwechsels im Bereich der Osteolysen und der knöchernen Destruktionen beobachtet werden.

\section{Stadium I}

- akute Entzündung, mit Infektion verwechselbar

- Rötung, Überwärmung, teils erhebliche Schwellung

- Röntgen: Demineralisierung, Fragmentierung, ggf. in der Frühphase noch keine knöchernen Veränderungen

\section{Stadium II}

Das Stadium II weist auffallende Reparationsvorgänge auf. Der fragmentierte Knochen wird abgerundet, umgebaut oder resorbiert. Die initial oft monströse Weichteilschwellung klingt allmählich ab. Es zeigen sich nun deutliche, allerdings unharmonische, knöcherne Heilungstendenzen, wobei typischerweise an den Knochenenden eine Sklerosierung und gelenknah eine Demineralisierung des Knochens festzustellen ist. Die sich zuspitzenden Knochenenden können das Auftreten eines Malum perforans mit entsprechenden Hautschädigungen verursachen, infolge sich bakterielle Infektkomplikationen einstellen können.

Stadium II

- Rückgang der Entzündungszeichen

- Röntgen: Resorption knöcherner Fragmente, Knochenneubildung, Frakturheilung

\section{Stadium III}

Im Stadium III kommt es durch eine Revaskularisierung zu Remodellierungsvorgängen, wobei diese teilweise in erheblichen Gelenkdeformierungen enden. Die Knochenenden spitzen sich $\mathrm{zu}$, werden sklerotischer und bieten die so genannte „Candy Stick“-Deformität (Anblick eines „abgelutschten Zuckerstengels“).

Stadium III

- Abklingen der Entzündungszeichen, geringe Schwellenneigung

- Röntgen: knöcherne Remodellierung, knöcherner oder fibröse Gelenkversteifungen (Ankylosen) 


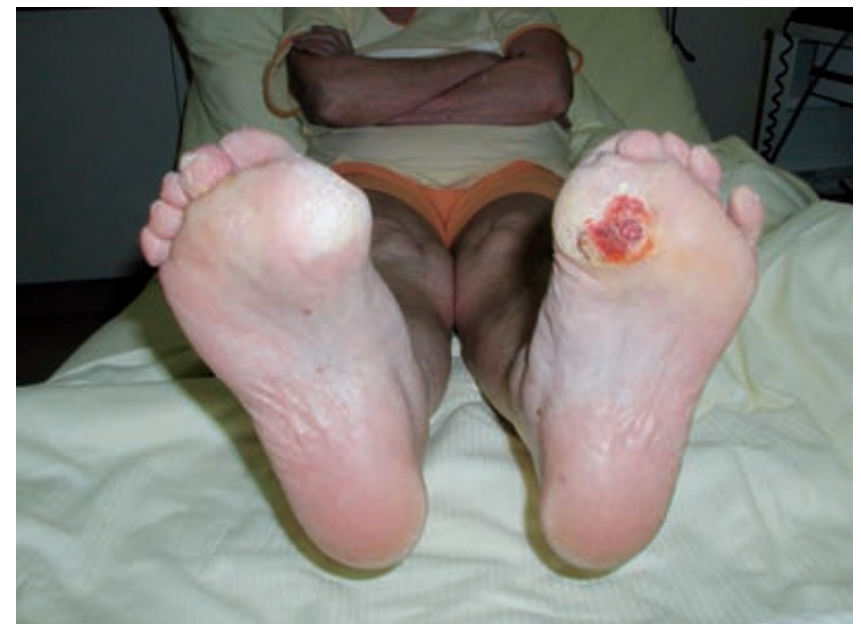

Abb. 1 Malum perforans durch lokale Überlastung oder durchspießende Knochenfragmente bei neuropathischem Sensibilitätsverlust.

\section{Klassifikation nach Sanders}

Für die Lokalisationszuordnung ist die Klassifikation von Sanders gebräuchlich, die die diabetisch-neuropathische Osteoarthropathie, gemäß der Stadieneinteilung, in 5 Hauptgruppen einordnet:

\section{Typ I}

Beim Typ I imponiert die spitz zulaufende Zuckerstengel-Form der Mittelfußknochen als Ausdruck einer bereits einsetzenden Reabsorption der Knochenstrukturen. Geprägt wird dieses Stadium durch das plantare Durchbiegen der Mittelfußknochen und einen resultierenden zentralen Malum perforans. Begleitet werden diese Veränderungen von hinzutretenden Spreizfuß- und Zehendeformitäten (Abb.1).

Typ II

Beim Typ II sind die Tarso-metatarsalGelenke (Lisfranc-Gelenk) betroffen, wodurch es zu einer empfindlichen Störung der Rückfußstatik kommt. Es resultiert hieraus eine vermehrte Druckbelastung des Cuneiforme-naviculare-Gelenks der Fußwurzel mit zwangsläufiger Entstehung eines Malum perforans (Abb.2).

\section{Typ III}

Der Typ III lässt eine Knochenreabsorption vornehmlich im Bereich des Chopart-Gelenks in Höhe des Talus und Fußkahnbeines beobachten. Der Rückfuß nimmt hier eine zunehmende Spitzfußstellung ein, wodurch der Vorfuß sich hackenfüßig aufstellt. Dieses Phänomen wird auch als diabetischer Schaukelfuß bezeichnet. Geprägt ist dieses Stadium durch das völlige Einbrechen des Fußlängsgewölbes, wodurch eine Drucküberlastung im mittleren Bereich der Fußsohlen resultiert. Hierdurch tritt eine Subluxation des Talus ein, die im Einzelfall in einer direkten Fußsohlen-Perforation gipfeln kann.

Typ IV

Der Typ IV ist eher selten anzutreffen und ist geprägt durch Veränderungen am oberen Sprunggelenk. Ebenso selten treten Knochenreabsorption im Bereich des hinteren unteren Sprunggelenks (talokalkaneares Gelenk) auf. In der Regel sind hier Tibia und Talus betroffen. Der instabil werdende Rückfuß kippt varisch um und verschiebt sich nach medial (Bajonettstellung).

\section{Typ $V$}

Eine weitere Knochensinterung führt $\mathrm{zu}$ einer Destruktion des Fersenbeins, die in eine Rückfußverplumpung, dem Typ V, mündet. Hierdurch resultiert eine verstärkte Druckbelastung auf den Mittelund Vorfuß. Die knöchernen Destruktionen führen nicht selten zur Ausbildung eines therapieresistenten Malum perforans.

Neben den destruierenden Prozessen am Fußskelett werden immer häufiger auch Osteoarthropathie z.B. am Kniegelenk als schwerwiegende Folge des Diabetes mellitus erkannt.

Hierbei kommt es, ohne dass ein adäquates Trauma vorausging, zu einem schmerzlosen Sintern tragender Gelenkflächen. Eine zunehmende Fehlstellung oder Funktionsminderung des betrof-

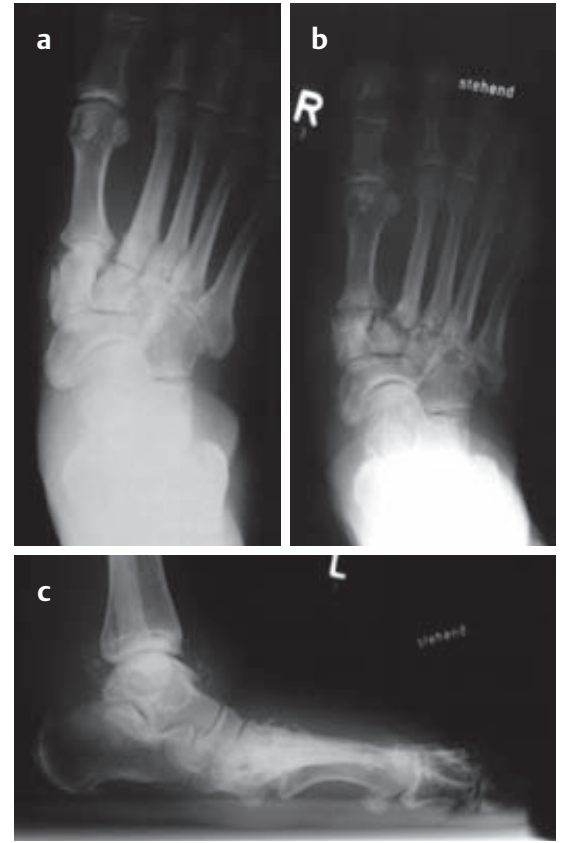

Abb.2a-c Charkot-Destruktion Typ II mit sekundärer Knick-Plattfußbildung und Gefahr eines zentralen Malum perforans. Progredienz von Stadium I (a) zu Stadium III (b) innerhalb von 10 Monaten.

fenen Gelenkes führen letztendlich in die weiterführende Diagnostik. Je nach Stadium kann der Prozess in einer vollständigen Zerstörung der anatomischen Gelenksstrukturen führen.

\begin{tabular}{|ll}
\hline Tab.3 & Klassifikation nach Sanders \\
\hline Typ & Lokalisation \\
\hline I & $\begin{array}{l}\text { Zehen und Vorfuß (Metatarso- } \\
\text { phalangealgelenke) }\end{array}$ \\
\hline II & $\begin{array}{l}\text { Lisfranc-Gelenk (kleine Fußwurzel- } \\
\text { knochen) }\end{array}$ \\
\hline III & Chopart-Gelenke \\
\hline IV & oberes Sprunggelenk \\
\hline V & $\begin{array}{l}\text { Fersenbein und unteres Sprung- } \\
\text { gelenk (Subtalargelenk) }\end{array}$ \\
\hline
\end{tabular}

\section{Behandlungsmöglichkeiten}

\section{Stadium I und II nach Eichenholtz}

Eine optimale schuhorthopädische Versorgung bei intakter Haut sowie entlastende Unterschenkel-Orthesen bei Ulzera stellen als konservative Behandlungsformen die Therapie der Wahl bei der Osteoarthropathie im Stadium I (bis II) nach Eichenholtz dar. Ziel ist es, aufgrund der eingeschränkten Sensibilität mechanischen Druck zu vermeiden. 
Die beste Therapie der Osteoarthropathie ist das Verhindern eines Ulkus durch optimale Schuhversorgung und Fußpflege.

Bei vorliegenden Destruktionen im Rückfußbereich sollte zudem bei der in der Regel zu beobachtenden Adipositas eine deutliche Gewichtsreduktion angestrebt werden.

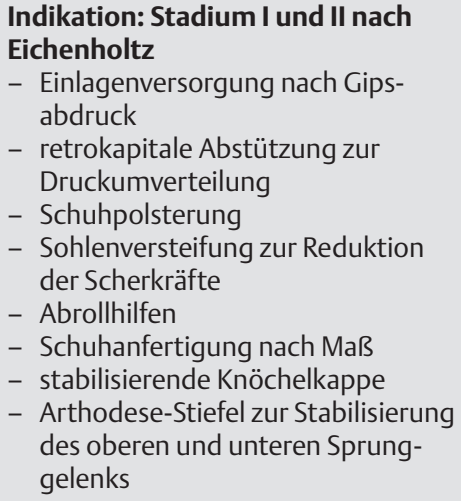

Ziel:

- Ruhigstellung

- Traumaprophylaxe

- Reduktion der Weichteilschwellung

- Optimierung der Druckverteilung und damit Ulkus-Prophylaxe

Kontraindikationen:

- tief greifende Weichteileinfekte, Osteiitiden

- schlechte Hautqualität

- schlechte Patientencompliance

- bestehendes Fersenulkus

\section{Operationsindikationen}

Ein Malum perforans sollte dann einer operativen Sanierung zugeführt werden, wenn es tiefer als sein Durchmesser ist und knöcherne Strukturen mitbeteiligt sind. Floride Weichteileinfektionen werden je nach Ausdehnung operativ saniert.

Bei zunehmenden Instabilitäten, Deformierung bei knöchernem Substanzverlust und letztlich Luxation von Gelenken bzw. knöchernen Strukturen ist ebenfalls eine operative Intervention anzustreben.
Operationsindikationen
- tief reichende Ulzera
- Knochenbeteiligung
- Instabilitäten, Deformitäten und Luxationen
- Weichteilinfektionen
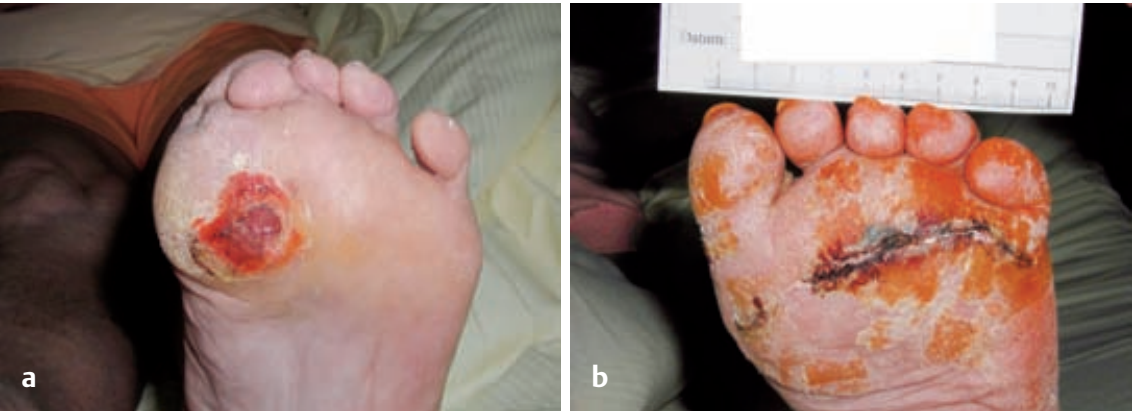

Abb. 3a, b Das Malum perforans ist grundsätzlich kontaminiert und meist nur chirurgisch zur Ausheilung zu bringen. Nach lokaler Excision und Sequestrektomie kann nach Abheilung - sofern erforderlich - die Wiederherstellung des Alignements zur Verbesserung der Fußabwicklung vorgenommen werden. Dies wurde hier durch eine Resektionsarthroplastik der Metatarsaleköpfchen und Weichteilkorrektur erreicht.

Das operative Konzept sieht vornehmlich eine operative Sanierung von Druckulzera sowie die Resektion prominenter und zerstörter Knochen (knöchernes Debridement) vor. Tief greifende Weichteilinfektionen werden entlastet und ggf. Septopalketten für bis zu zwei Wochen eingelegt (Abb.3).

Sekundäre luxierte Gelenke werden den Erfordernissen nach reponiert und operativ stabilisiert, wodurch die Ausheilung einer aktiven Osteoarthropathie nachweislich begünstigt werden kann.

Von Bedeutung ist die Mittelfußknochenresektion nach Velpeau und Baumgartner, die eine Ausheilung der Ulzera nach durchschnittlich sechs Wochen erwarten lässt.

Überstehende, ulkusgefährdete Knochenvorsprünge werden sparsam reseziert. Als günstigster Operationszeitpunkt hat sich hierbei das Stadium III nach Eichenholtz erwiesen.

Für die erforderliche Ruhigstellung bietet sich zum Beispiel die Montage eines Hoffmann-Fixateur für sechs bis acht Wochen an. Im amerikanischen Sprachraum wird ab dem 1 . bzw. 2. postoperativen Tag die Anlage eines so genannten Total-Contakt-Cast empfohlen.

Der günstigste Operationszeitpunkt ist das Stadium III nach Eichenholtz.

\section{Operationsindikation unter dem Gesichtspunkt der Lokalisation}

\section{Typ I nach Sanders}

Sofern vertretbar und nicht durch eine Weichteil- und Knocheninfektion er- schwert, wird das aktive Stadium der Osteoarthropathie (Eichenholtz Stadium I bis II) abgewartet.

Als operative Maßnahmen bieten sich je nach Befundausprägung und drohendem Malum perforans Resektionsarthroplastiken einzelner destruierter Mittelfußknochen ohne Zehenamputationen bis an die Grenze des Chopart-Gelenkes an. Es sollte darauf geachtet werden, möglichst zwei Mittelfußstrahlen zu erhalten, um eine adäquate orthopädische Schuhzurichtung zu gewährleisten.

Nach der definitiven Ausheilung sollte das Schuhwerk eine Abrollhilfe und Sohlenversteifung neben einer queren retrokapitalen Abstützung erhalten.

\section{Typ II}

Beim Typ II sind aufwändige rekonstruktive Operationsansätze wegen der schlechten Knochenqualität nicht Erfolg versprechend. Die operativen Eingriffe sind im Wesentlichen als Prophylaxe und Therapie von Weichteilkomplikationen (Malum perforans) anzusehen. Unter Schonung der tragenden Fußsohle können prominente und spitze Knochenfragmente abgetragen werden.

Die anschließende Behandlung sieht die temporäre Anlage eines Vollkontakt-Unterschenkelgehgips- bzw. -kunststoffverbandes (Total-Contact-Casts) oder einer Orthese bis zur definitiven Versorgung der orthopädischen Schuhversorgung nach abgeschlossener Wundheilung und knöcherner Konsolidierung bzw. fibröser Ankylosierung (Scheingelenkbildung) vor. 

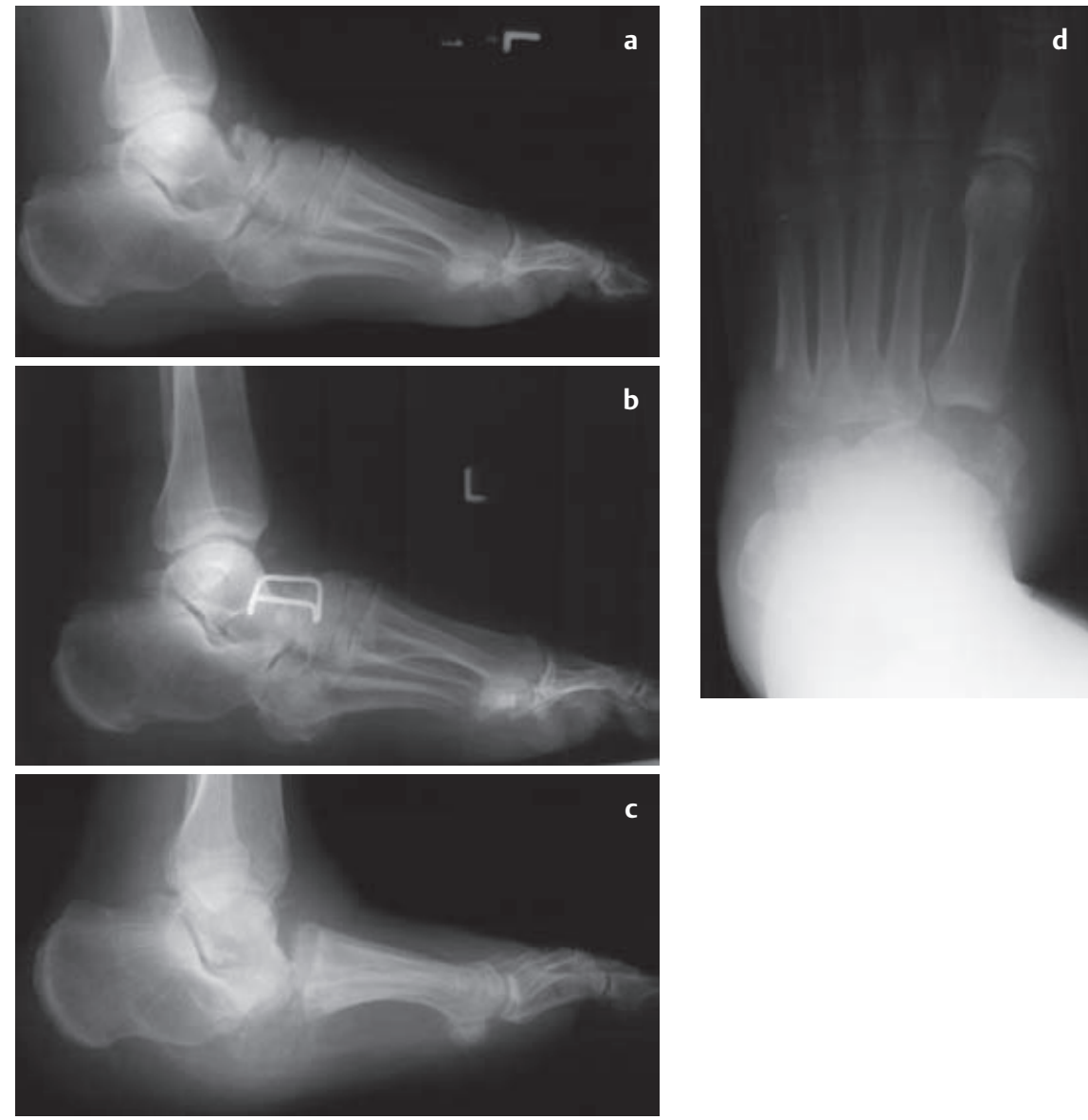

Abb. $4 a-d$

a Charcot. Destruktion Typ III, Stadium 2 (keine OP-Indikation).

b Fehlgeschlagene Talo-Naviculare-Arthrodese, drohendes zentrales Malum perforans.

c Z.n. Resektionsarthroplastik des Chopart-Gelenkes ohne Sicherung des Rückfußes. Trotz konsekutivem Talus verticalis akzeptable Funktion bei plantigrader Auftrittsfläche.

d Z.n. Resektion der Oss. cuneiformia bei Charkot Typ II nach konsolidierter Rückfußarthrodese. Ausbildung einer fibrösen Ankylose bzw. Nearthrose mit guter Funktion.

\section{Typ III}

Beim Typ III können, ebenso wie zuvor beschrieben, störende, überragende Knochenfragmente abgetragen werden.

Von entscheidender Bedeutung sind allerdings eine adäquate orthopädische Schuhversorgung und die konsequente Fußpflege von Seiten des Patienten (Abb.4).

Typ IV

Das therapeutische Konzept sieht beim Typ IV eine operative Resektion des oberen Sprunggelenkes über einen lateralen Zugang vor. Die distale Fibula (Außenknöchel) wird abgesetzt und eine horizontale Gelenkflächenresektion des oberen Sprunggelenkes vorgenommen. Die temporäre Fixierung gelingt mittels Anlage eines gelenküberschreitenden Fixa- teur externe von der distalen Tibia auf den Kalkaneus und den Vorfuß über ca. 6 Wochen. Je nach knöcherner Destruktion kann es ggf. auch notwendig werden, das gesamte Sprungbein zu resezieren. Danach kann in einer Unterschenkelorthese der sukzessive Belastungsaufbau eingeleitet werden. Es bildet sich bei günstigem Verlauf ein belastungsfähiges Scheingelenk (fibröse Ankylose) aus. Alternativ besteht die Möglichkeit der definitiven Arthodese des oberen Sprunggelenkes.

\section{Typ V}

Die konservative Behandlung des Typ V sieht initial zunächst eine Fersenbettung sowie den Ausgleich des Höhenverlustes im Fersenbereich durch Anpassung einer Unterschenkelorthese und später maßgerechte Anfertigung von orthopädischem Schuhwerk vor.
Die operativen Ansätze orientieren sich am Schweregrad der knochendestruktiven Veränderungen des Fersenbeins. Neben der fersenschonenden Abtragung knöcherner prominenter Knochenanteile werden osteonekrotische bzw. bei chronischen Fersenulkus osteomyelitische Herde ausgeräumt. Bei erheblicher Destruktion des Kalkaneus ggf. auch unter Einbeziehung des Talus werden diese reseziert und bis zur definitiven Wundkonsolidierung im Fixateur externe ruhig gestellt. Im Anschluss erfolgt die Anpassung einer Unterschenkelorthese mit entsprechendem Beinlängenausgleich. Im Einzelfall kann ein chronisch-therapieresistentes Fersenulkus durch eine plastisch-chirurgische Deckung (z.B. mithilfe eines freien Latissimus-dorsiLappens) zur Ausheilung gebracht werden.

\section{Amputationen}

Bei vollständigen, nicht mehr tragfähigen Knochendestruktionen sowie nicht beherrschbaren Weichteil- und Knocheninfektionen bleibt als Ultima Ratio oftmals nur noch eine Amputation.

Amputationen
- Zehen
- Keilresektionen eines infizierten
Strahls
- basisnahe Amputationen von Mittel-
fußknochen
- Resektion der Tarsalknochen (Opera-
tion nach Velpeau und Baumgartner,
Operation nach Link-Witzel) bzw. Me-
tatarsalknochen unter Belassen der
distalen Knochen (innere Fußampu-
tation)
- Amputation im Lisfranc-Gelenk
- Amputation im Chopart-Gelenk
- Resektion des Talus und Einstellen
des Kalkaneus unter die Tibia (nach
Pirogoff-Spitzy)
- Exartikulation des oberen Sprungge-
lenkes (nach Syme)

\section{Zusammenfassung}

Bei der Charcot-Arthropathie besteht zunächst das Problem der frühzeitigen Diagnosestellung. Die meisten Fälle werden verspätet im Verlauf der typischen, weitgehend schmerzfreien Destruktion und teils grotesker Deformierung tragender Gelenke, vor allem am Fuß, entdeckt.

Die Differenzierung des äußeren Erscheinungsbildes von Lymphödemen, Thrombose und Entzündung sowie anhand labortechnischer und bildgebender Befunde von arthrotischen Prozessen, (patholo- 
gischen) Frakturen anderer Genese, Osteomyelitis und Rheuma ist oft schwierig.

Beobachtungen klinischer und radiologischer Behandlungsverläufe der diabetisch-neuropathischen Osteoarthropathie lassen den Schluss zu, dass das frühzeitige Erkennen bzw. die Einleitung präventiver Maßnahmen das Krankheitsbild insofern günstig beeinflussen, dass ausgeprägter Fehlstellungen der betroffenen Gelenke deutlich gemildert werden können. Die konsequente, frühestmögliche und stadiengerechte Behandlung ist für den Therapieerfolg entscheidend. Wesentlicher Gesichtspunkt ist der Erhalt einer funktionstüchtigen Extremität.

Die adäquate Therapie wird kontrovers diskutiert. Orthopädietechnische Maßnahmen stehen im Vordergrund, progredient instabile Fehlstellungen mit Invalidisierung und unter konservativer Therapie persistierende Ulzera erfordern operative Maßnahmen. Diese bestehen hauptsächlich aus Resektionsarthroplastiken, vor allem der Mittelfußknochen, und stabilisierenden Achskorrekturen. Dabei sind alle gängigen Osteosyntheseverfahren problematisch. Die interne Osteosynthese lässt auch bei ausgebrannter Osteoarthropathie keine sichere knöcherne Konsolidierung erwarten. Es besteht die Gefahr von Implantatbruch,
Auslockerung und Durchspießung. Fixateur externe und Steinmann-Nägel haben eine hohe Gefahr von lokalen Infekten.

Angestrebt wird eine frontal lotgerechte Stellung mit plantigrader Belastbarkeit ohne Ulkusgefährdung bei fibröser Ankylose der betroffenen Gelenksabschnitte.

Auch autologe Spongiosaanlagerung oder Allografts lassen keine sichere knöcherner Fusion erwarten. Operative Maßnahmen können prinzipiell in allen Stadien durchgeführt werden, ein Abwarten des „Ausbrennens“ des Destruktionsprozesses (Stadium III nach Eichenholtz) ist für stabilisierende Achskorrekturen mit einem deutlich geringeren Risiko für Wundheilungsstörungen behaftet.

Eine sinnvolle Kombination konservativer und operativer Behandlungsmaßnahmen kann die Lebensqualität ganz erheblich bessern. Sehr wichtig in diesem Zusammenhang ist eine enge $\mathrm{Zu}-$ sammenarbeit der weiterbehandelnden Orthopäden und Chirurgen mit dem Orthopädie-Schumacher, die eine regelmäßige Kontrolle der hilfsmittelversorgten Patienten beinhalten muss. Eine adäquate schuhorthopädische Versorgung bzw. auch die Anlage eines Total-Contact-Casts ist - soweit vertretbar - die Therapie der Wahl.

\section{Literatur}

Bailey CC, Root HF. Neuropathic joint lesions in diabetes mellitus. J Clin Invest 1942; 21:649 Baumgartner R, Botta P. Amputation und Prothesenversorgung der unteren Extremität, 2. Aufl. Stuttgart: Enke 1995

Baumgartner R. Die orthopädische Versorgung des Diabetesfußes, Med Orthop Tech 1990; 110: $176-187$

Baumgartner R. Jean-Marie Charcot und sein Fuß. Med Orthop Tech 1998;118: 15-17

Charcot JM, Féré CH. Affection osseuse et articulicre du pied chez les diabétiques (pied diabétique). Arch Neurol 1883: 305-319

Eichenholtz SN. Charcot joints. Thomas, Springfeld/IL 1966

Jordan WR. Neuritic manifestations in diabetes. Arch Intern Med. 1936; 57: 307-366 Koller A, Fiedler R, Wetz HH. Der Fixateur externe zur Rekonstruktion der Fußstatik bei neurogener Osteoarthropathie. Orthopädie 2001; 30: 218-25

Pappa J, Myerson M, Girard P. Salvage, with arthrodesia in intractable diabetic neuropathic arthropathy of the foot and ankle. J Bone Joint Surg (Am) 1993; 75: 1056-1066

Sanders LJ, Frykberg RG. Diabetic neuropathic osteoarthropathy. In: Frykberg RG (Hrsg) The high risk foot in diabetes mellitus. New York: Livingstone 1991; 297-338

Thompson RC Jr, Clohisy DR. Deformity following fracture in diabetic neuropathic osteoarthropathy. J. Bone Joint Surg (Am) 1993; 75: 1765-1773

Wetz HH, Baumgartner R. Die orthopädischchirurgische Behandlung der diabetisch-neuropathischen Osteoarthropathie. Med Orthop Tech 1998; 118: 6-14

\section{Dr. med. Torsten Eyfferth}

Oberarzt der Abteilung für Unfallchirurgie und orthopädische Chirurgie Dr. med. Hans-Peter Abt Chefarzt der Abteilung für orthopädische und traumatologische Fußchirurgie Prof. Dr. med. Reinhard Hoffmann Ärztlicher Direktor

BG Unfallklinik Frankfurt am Main Friedberger Landstraße 430 60389 Frankfurt am Main 